\title{
Debated waters
}

\section{New experiments and computer simulations on how water behaves when it is supercooled are poised to rekindle long-standing debates.}

Whether water is a simple or complex liquid is not a matter of taste. Ice floats, liquid water at $4{ }^{\circ} \mathrm{C}$ expands regardless of whether it is heated or cooled, and below $33^{\circ} \mathrm{C}$ it becomes less viscous when compressed. These and many other anomalous behaviours, which remain far from being understood, are unlike those of any other liquid (although other tetrahedrally bonded liquids such as silicon share many anomalies with water). Also they are central to life as we know it: for example, aquatic life in freshwater lakes can survive in the winter because the bottom remains at about $4{ }^{\circ} \mathrm{C}$ while ice stays on the surface.

Yet water at ambient pressure can remain liquid down to $-44^{\circ} \mathrm{C}$ - the approximate temperature at which ice forms spontaneously and quickly — provided that it is kept away from impurities or perturbations such as mechanical vibrations ${ }^{1}$. Supercooled water, in which molecules arrange into a disordered network of imperfect tetrahedra, is thus thermodynamically unstable with respect to ice, where water molecules arrange in a more open (and thus less dense) perfectly tetrahedral network. However, stable forms of ice (which are made of tetrahedra packed in layers with either hexagonal or cubic symmetry) may not nucleate directly from the liquid. As Hajime Tanaka and colleagues describe on page 733 , computer simulations of classical models of water predict that hexagonal and cubic ices nucleate inside a precursor form of ice with tetragonal symmetry that is structurally more similar to liquid water than to the two ices. They also find that, for tetragonal ice, the temperature of spontaneous (homogeneous) ice nucleation depends on pressure in the same way as the melting temperature does, hence providing a link between kinetics (nucleation) and thermodynamics (melting). In a linked News \& Views on page 670 , Ben Slater and David Quigley provide further support for the likelihood of tetragonal ice being an energetic stepping stone to the crystallization of water: among common ice phases, quantum-mechanical density functional theory predicts that the energy of tetragonal ice is closest to that of the thermodynamically stable hexagonal ice.

Even at temperatures much higher than that at which ice spontaneously nucleates, supercooled water gets more ordered as it is cooled. And the way in which the ordering comes about has been postulated to be responsible for water's anomalous behaviour ${ }^{1}$. Indeed, the minimum in water's isothermal compressibility at $46^{\circ} \mathrm{C}$ (a direct consequence of the density maximum at $4{ }^{\circ} \mathrm{C}$ ) is thought to signal the possible presence of a singularity (a liquid-liquid critical point) where the compressibility (and other properties) would diverge. Unfortunately, the singularity would be buried at temperatures below that of water's kinetic stability limit, and thus be impossible to reach experimentally. However, computer simulations of some coarse-grained water models have predicted a transition between two forms of liquid water whose density difference vanishes at such critical point ${ }^{2}$.

The debate about whether such a liquidliquid transition and associated critical point exist has been ongoing for a few decades ${ }^{1-3}$, and new work promises to reignite it. By using advanced Monte Carlo computational methods, David Limmer and David Chandler have argued since 2011 that in all atomistic models of water the apparent liquid-liquid transition is instead a liquid-ice transition ${ }^{4}$. Now, Pablo Debenedetti and collaborators report in Nature that state-of-the-art freeenergy calculations show unambiguously that

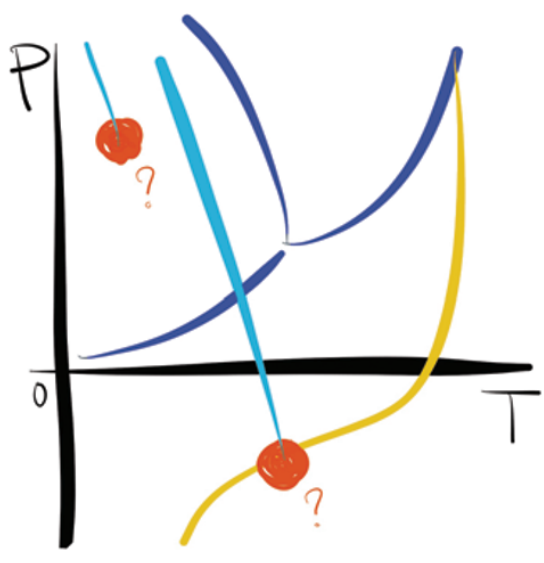

Figure 1 | New clues reignite the debate about the existence and location of a presumed liquid-liquid transition (light blue) and associated critical point (red dots) in supercooled water. This artistic impression of water's pressure $(P)$-temperature ( $T$ ) phase diagram also includes the transitions between the vapour, liquid and gas phases (dark blue), and the stability limit of liquid water with respect to its vapour (yellow). a long-used model of water (ST2) exhibits a discontinuous liquid-liquid transition ${ }^{5}$.

Still, predictions from most, if not all, water models have been shown to deviate from experimental data in one way or another. Also writing in Nature, Anders Nilsson and colleagues show that the ordering of supercooled water, determined by measuring the patterns of X-ray laser pulses scattered by water microdroplets, rises continuously as temperature drops down to just below that at which ice spontaneously nucleates (below the nucleation temperature, a few microdroplets remained liquid for about a millisecond), indicating the absence of a discontinuity at ambient pressure ${ }^{6}$. As Alan Soper argues in a related News \& Views on page 671, these experiments do not preclude the existence of a liquid-liquid critical point, but will make it tougher to search for it.

Yet measurements of the velocity of sound across microlitre volumes of liquid water under tension (that is, at negative pressures, which can be achieved by supercooling at conditions where cavitation can be avoided) and computer simulations of a popular water model (TIP4P/2005) are compatible with thermodynamic scenarios for which the isothermal compressibility shows a maximum ${ }^{7}$, now claim Frédéric Caupin and co-workers in the Proceedings of the National Academy of Sciences of the USA. This is in fact the case of a liquid-liquid scenario with a critical point at positive pressure (Fig. 1). However, in a News \& Views on page 673, Austen Angell writes that Caupin and colleagues' measurements suggest instead that, as the water was supercooled at constant volume, it continuously transformed into a liquid phase with lower tension, a situation that corresponds to a liquid-liquid transition associated with an alternative scenario in which the critical point lies deep at negative pressures (Fig. 1).

The waters are indeed nowhere close to clearing up.

\section{References}

1. Debenedetti, P. G. J. Phys. Condens. Matter 15, R1669-R1726 (2003).

2. Poole, P. H., Sciortino, F., Essmann, U. \& Stanley, H. E. Nature 360, 324-328 (1992).

3. Ball, P. $\mathrm{H}_{2} \mathrm{O}:$ A Biography of Water (Phoenix, 2000).

4. Limmer, D. T. \& Chandler, D. J. Chem. Phys. 135, 134503 (2011).

5. Palmer, J. C. et al. Nature 510, 385-388 (2014).

6. Sellberg, J. A. et al. Nature 510, 381-384 (2014)

7. Pallares, G. et al. Proc. Natl Acad Sci. USA 111, 7936-7941 (2014). 